\title{
Short-Chain Fatty Acids Improve Poststroke Recovery via Immunological Mechanisms
}

\author{
Rebecca Sadler, ${ }^{1,2}$ Julia V. Cramer, ${ }^{1}$ Steffanie Heindl, ${ }^{1}$ Sarantos Kostidis, ${ }^{3}$ Dene Betz, ${ }^{4}$ OKielen R. Zuurbier, ${ }^{4}$ \\ ĐBernd H. Northoff, ${ }^{5}$ Marieke Heijink, ${ }^{3}$ Mark P. Goldberg, ${ }^{4}$ Erik J. Plautz, ${ }^{4}$ @Stefan Roth, ${ }^{1}$ Rainer Malik, ${ }^{1}$ \\ (D)Martin Dichgans, ${ }^{1,2}$ Lesca M. Holdt, ${ }^{5}$ Corinne Benakis, ${ }^{1}$ Martin Giera, ${ }^{3}$ Ann M. Stowe, ${ }^{4,6}$ and Arthur Liesz ${ }^{1,2}$ \\ ${ }^{1}$ Institute for Stroke and Dementia Research, University Hospital, Ludwig-Maximilians-University LMU, 81377, Munich, Germany, ${ }^{2}$ Munich Cluster for \\ Systems Neurology (SyNergy), 81377, Munich, Germany, ${ }^{3}$ Leiden University Medical Center, Center for Proteomics \& Metabolomics, 2300 RC Leiden, The \\ Netherlands, ${ }^{4}$ Department of Neurology and Neurotherapeutics, Peter O’Donnell Jr. Brain Institute, University of Texas Southwestern Medical Center, \\ Dallas, Texas 75390, ${ }^{5}$ Institute of Laboratory Medicine, University Hospital, Ludwig-Maximilians-University LMU, 81377, Munich, Germany, \\ and ${ }^{6}$ Department of Neurology, University of Kentucky, Lexington, Kentucky 40508
}

Recovery after stroke is a multicellular process encompassing neurons, resident immune cells, and brain-invading cells. Stroke alters the gut microbiome, which in turn has considerable impact on stroke outcome. However, the mechanisms underlying gut- brain interaction and implications for long-term recovery are largely elusive. Here, we tested the hypothesis that short-chain fatty acids (SCFAs), key bioactive microbial metabolites, are the missing link along the gut- brain axis and might be able to modulate recovery after experimental stroke. SCFA supplementation in the drinking water of male mice significantly improved recovery of affected limb motor function. Using in vivo wide-field calcium imaging, we observed that SCFAs induced altered contralesional cortex connectivity. This was associated with SCFA-dependent changes in spine and synapse densities. RNA sequencing of the forebrain cortex indicated a potential involvement of microglial cells in contributing to the structural and functional remodeling. Further analyses confirmed a substantial impact of SCFAs on microglial activation, which depended on the recruitment of $T$ cells to the infarcted brain. Our findings identified that microbiota-derived SCFAs modulate poststroke recovery via effects on systemic and brain resident immune cells.

Key words: microbiome; neuroinflammation; plasticity; stroke models

\section{Significance Statement}

Previous studies have shown a bidirectional communication along the gut- brain axis after stroke. Stroke alters the gut microbiota composition, and in turn, microbiota dysbiosis has a substantial impact on stroke outcome by modulating the immune response. However, until now, the mediators derived from the gut microbiome affecting the gut-immune-brain axis and the molecular mechanisms involved in this process were unknown. Here, we demonstrate that short-chain fatty acids, fermentation products of the gut microbiome, are potent and proregenerative modulators of poststroke neuronal plasticity at various structural levels. We identified that this effect was mediated via circulating lymphocytes on microglial activation. These results identify short-chain fatty acids as a missing link along the gut-brain axis and as a potential therapeutic to improve recovery after stroke.

\section{Introduction}

Stroke induces a multiphasic pathophysiological cascade, which consists of an initial excitotoxicity followed by a longer neuroin-

Received June 12, 2019; revised Nov. 25, 2019; accepted Dec. 3, 2019.

Author contributions: R.S., J.V.C., S.K., D.B., K.R.Z., B.H.N., M.H., E.J.P., and S.R. performed research; R.S., J.V.C., S.H., S.K., K.R.Z., B.H.N., M.P.G., E.J.P., R.M., M.D., L.M.H., C.B., M.G., A.M.S., and A.L. analyzed data; R.S. wrote the first draft of the paper; S.H., S.K., B.H.N., M.P.G., E.J.P., M.D., L.M.H., C.B., M.G., and A.M.S. edited the paper; S.R., A.M.S., and A.L. designed research; A.L. wrote the paper.

This work was supported by the German Research Foundation (Deutsche Forschungsgemeinschaft, LI2534/2-1), the European Research Council (ERC-StG 802305), the Vascular Dementia Research Foundation, and the Munich Cluster for Systems Neurology (EXC 2145 SyNergy) ID 390857198 to A.L., the Texas Institute for Brain Injury and flammatory phase within the brain (Dirnagl et al., 1999; Iadecola and Anrather, 2011). Moreover, stroke can be regarded as a systemic disease affecting also remote organ function, including the lung (Austin et al., 2019), heart (Bieber et al., 2017), and immune system and intestinal function (Singh et al., 2016). Recently, it has been shown that a dysbiotic gut microbiota is correlated with a

Repair to M.P.G. and A.M.S., and National Institutes of Health/National Institute of Neurological Disorders and Stroke (NS088555) to A.M.S. We thank Kerstin Thu $\beta$-Silczak for excellent technical assistance.

The authors declare no competing financial interests.

Correspondence should be addressed to Arthur Liesz at Arthur.Liesz@med.uni-muenchen.de.

https://doi.org/10.1523/JNEUROSCI.1359-19.2019

Copyright $\odot 2020$ the authors 
worsened outcome in patients (Xia et al., 2019), and that these changes are evident up until 3 weeks after hospitalization (Swidsinski et al., 2012). We have previously demonstrated an important role of the gut microbiome on stroke outcome in proof-of-principle experiments using germ-free and recolonized mice (Singh et al., 2018). Further experimental studies in rodent stroke models have identified a key role for the immune system, particularly braininvading lymphocytes originating from the intestinal immune compartment, in mediating along the gut-brain axis (Benakis et al., 2016; Singh et al., 2016).

The gut microbiome produces a large number of bioactive metabolites which may affect brain function via modulating the immune system or afferent neuronal pathways (Kau et al., 2011; Cryan and Dinan, 2012). In particular, the metabolite group of short-chain fatty acids (SCFAs) acetate, butyrate, and propionate have been shown to readily cross the blood-brain barrier (Frost et al., 2014; Morrison and Preston, 2016) and affect brain function in development, health, and disease. For example, SCFA treatment improved the disease course in experimental autoimmune encephalitis by promoting anti-inflammatory mechanisms and reducing axonal damage (Haghikia et al., 2015). In mouse models of chronic stress, mice that received SCFA treatment exhibited significant improvements in antidepressant and anxiolytic behaviors, which was accompanied by reduced plasma corticosterone levels and differential gene regulation (van de Wouw et al., 2018).

More recently, the role of SCFAs in modulating the immune system has been studied in great detail. Through these investigations, they have been shown to play a role in the polarization of $\mathrm{T}$ cells in the intestinal immune compartment and inducing antiinflammatory T-cell subset (Smith et al., 2013; Tan et al., 2016). Other studies have shown a critical role for microbiota-derived SCFAs in the maturation of microglial cells, the brain's resident immune cells (Erny et al., 2015). However, SCFAs can have farreaching pleiotropic effects also beyond the immune system, including a direct effect on neuronal function through their potent function as histone deacetylase inhibitors (Bourassa et al., 2016). Accordingly, the importance of SCFA function has been implicated in neurodegenerative diseases and even in postischemic neurogenesis (Chuang et al., 2009; Kim et al., 2009).

Despite the key contribution of the gut microbiome to stroke outcome and the identification of SCFA as one of the microbiome's primary bioactive mediators, the role of SCFAs and their potential therapeutic use for poststroke recovery in the chronic phase after brain ischemia have not yet been investigated. In this study, we comprehensively investigated the effect of SCFA administration on poststroke recovery using advanced behavior analyses, in vivo wide-field calcium imaging, transcriptomic studies, and histological analyses to study and link SCFA-mediated recovery mechanisms from the molecular level up to behavior.

\section{Materials and Methods}

Animals and treatment. All experimental protocols were approved by the responsible governmental committees (Regierung von Oberbayern, Munich, Germany and Institutional Animal Care and Use Committee, University of Texas Southwestern, Dallas). Specific pathogen-free C57BL/6J female and male mice were purchased from Charles River Laboratories or The Jackson Laboratory. On the day of arrival, mice were $6-8$ weeks of age. Male mice were used for all experiments, except for analysis of dendritic spine densities (see Fig. $2 A-F$ ), in which female mice were used. Mice were given SCFAs ( $25.9 \mathrm{~mm}$ sodium propionate, $40 \mathrm{~mm}$ sodium butyrate, and $67.5 \mathrm{~mm}$ sodium acetate) or salt-matched control (133.4 mm sodium chloride) (9265.1, ROTH) in drinking water ad libitum for 4 weeks as previously reported (Smith et al., 2013; Erny et al.,
2015). Mice were continuously given supplemented water until the end of the experiment. For antibiotic treatment, mice received metronidazol $0.5 \mathrm{mg} / \mathrm{ml}$ (Millipore Sigma, \#46461), neomycin $0.5 \mathrm{mg} / \mathrm{ml}$ (Millipore Sigma, \# N1142), ampicillin $0.5 \mathrm{mg} / \mathrm{ml}$ (Millipore Sigma, \#31591), and vancomycin $0.5 \mathrm{mg} / \mathrm{ml}$ (Millipore Sigma, \#V8138) and with 5\% sucrose in drinking water. All drinking water solutions were prepared and changed twice a week and blinded for experimenters.

Experimental stroke model. For the photothrombotic stroke model (PT), mice were anesthetized with isoflurane, delivered in a mixture of $30 \% \mathrm{O}_{2}$ and $70 \% \mathrm{~N}_{2} \mathrm{O}$. Mice were placed into a stereotactic frame; and throughout the surgical procedure, body temperature was maintained at $37^{\circ} \mathrm{C}$ with a mouse warming pad. Dexpanthenol eye ointment was applied to both eyes. A skin incision was used to expose the skull. Bregma was located and using the laser the lesion location was marked in the left hemisphere (1.5 mm lateral and $1.0 \mathrm{~mm}$ rostral to bregma). Mice were then injected intraperitoneally with Rosa Bengal (Millipore Sigma, \#198250). Shielding was placed on the skull, allowing a 2-mm-diameter circular exposure over the lesion area. After $10 \mathrm{~min}$, the laser (Cobolt Jive, $561 \mathrm{~nm}, 25 \mathrm{mV}$ output power with fiber collimation at $\mathrm{f}=7.66 \mathrm{~mm}$ ) was applied to the lesion area for $17 \mathrm{~min}$.

For the distal, permanent occlusion of the middle cerebral artery (dMCAo), the distal middle cerebral artery (MCA) was permanently electrocoagulated as previously described (Llovera et al., 2014). In brief, under isoflurane anesthesia, mice were placed in the lateral position. A skin incision was made to expose the skull. Using a drill, a burr hole into the skull revealed the bifurcated MCA. Using electric forceps, the artery was occluded and checked for no blood flow. Mice were then sutured across the skull incision.

For the filament, transient occlusion of the MCA (fMCAo), the internal carotid artery was transiently occluded for $60 \mathrm{~min}$ as previously described (Singh et al., 2016). In brief, mice were anesthetized with isoflurane, and an incision was made to expose the temporal bone. A laser doppler probe was affixed to the MCA territory to determine blood flow. Mice were included when a $>80 \%$ cerebral blood flow drop was induced. At the neck, an incision to the skin exposed the common carotid and external carotid artery, which were ligated. The filament was inserted into the internal carotid artery for $60 \mathrm{~min}$, and the MCA was occluded. Mice were sutured across the neckline. In total, 13 mice per group were used. In the control group, 8 mice died. In the SCFA-supplemented group, 3 mice died and 3 mice had no quantifiable infarct.

After all surgeries, mice were recovered in a nursing box at $37^{\circ} \mathrm{C}$ for $15 \mathrm{~min}$ and then returned to home cages.

Wide-field calcium imaging. In vivo wide-field calcium imaging was performed as previously published (Cramer et al., 2019). Briefly, we used Thy1GCaMP6s heterozygous reporter mice. Mice were scalped, and transparent dental cement was placed upon the intact skull at least $3 \mathrm{~d}$ before the start of the experiment. Resting-state in vivo imaging was performed under mild anesthesia $(0.5 \mathrm{mg} / \mathrm{kg}$ body weight of medetomidin with $0.75 \%$ isoflurane inhalation). Mice were placed in a stereotactic frame below a customized macroscopic imaging setup, and the mouse cortex was illuminated with $450 \mathrm{~nm}$ blue LED light. We recorded for 4 min in resting state with a CCD camera at $25 \mathrm{~Hz}$ frame rate. Functional connectivity was computed for seed-based analysis with a seed pixel time series centered in the right caudal forelimb cortex, contralesional to the infarcted cortex. Pearson's correlation between the time course of this seed, and any other signal time course within the masked area was computed; then Fisher's $z$-transformed and topographically displayed. Analogous functional connectivity between seed pairs was calculated as correlation between signal time courses of two seeds within the sensorimotor cortex. Seeds were defined as previously described (Cramer et al., 2019). Mean correlation per group was calculated for each time point. We used two-sample $t$ test and Bonferroni correction for statistical testing.

Automated skilled reaching. Behavioral training and assessments were measured using an automated reach task previously described (Becker et al., 2016). Mice are trained to pull an isometric lever using the contralesional forelimb, with each successful application of force criteria rewarded by a drop of peanut oil. For the first week, mice were trained in groups of 23 littermates for $6 \mathrm{~h}$. During this time, spontaneous peanut oil deliveries occurred at randomly spaced time intervals ranging from $30 \mathrm{~s}$ 
to $5 \mathrm{~min}$. Additionally, the isometric handle was positioned $\sim 0.5 \mathrm{~cm}$ from the inner edge of the chamber during these $6 \mathrm{~h}$ sessions. The force criterion necessary to trigger a $4 \mu \mathrm{l}$ peanut oil droplet was set just above electrical noise so that any slight touch could trigger peanut oil dispersion. For the next 3 weeks, mice were trained individually for $2 \mathrm{~h}$ sessions. The handle was positioned further back at $1 \mathrm{~cm}$ from the inner edge of the chamber. The criterion for success during training sessions was changed based on a customized, adaptive MATLAB program. The adaptive program initially set the criterion for success at a specified value of at least $1 \mathrm{~g}$. After the first 15 pulls, the criterion was adjusted to the median force value. The adjustments continued for the duration of the session unless the median reached the maximum force requirement of $20 \mathrm{~g}$. Finally, we fixed the criterion at $20 \mathrm{~g}$ to measure baseline motor function for three consecutive sessions. After stroke, assessments of reaching behaviors were measured weekly for 6 weeks.

SCFA quantification. Mice were saline perfused, and organs extracted. For feces, colon content was collected; and for plasma, cardiac puncture blood was centrifuged at $4^{\circ} \mathrm{C}$ at $3000 \mathrm{rpm}$ and the plasma removed. All samples were frozen immediately on dry ice. SCFAs (acetic, propionic, and butyric acid) were determined according to published protocols, using gas chromatography-mass spectrometry (Hoving et al., 2018). Briefly, for plasma, $10 \mu \mathrm{l}$ was used and feces were homogenized with LC-MS grade water in a bead beater. Samples were spiked with deuterated internal standards for each SCFA. Subsequently, SCFAs were derivatized using pentafluorobenzyl bromide and analyzed using gas chromatography-electron capture negative ionization in single ion monitoring mode. Quantification was performed against external calibration lines.

Infarct volumetry. After saline perfusion, the brain was removed from the skull and placed immediately on dry ice. Microscopy slides were mounted with 20 - $\mu \mathrm{m}$-thick coronal cryosections cut at $400 \mu \mathrm{m}$ intervals for $\mathrm{dMCAo}$ and $\mathrm{AMCAo} \mathrm{lesions,} \mathrm{and} \mathrm{at} 120 \mu \mathrm{m}$ intervals for PT lesions. Sections were then stained with cresyl violet solution. For the infarct volume, all slides were scanned using $600 \mathrm{dpi}$. The area of infarct tissue was measured using the ImageJ software (National Institutes of Health) and integrated for calculation of infarct volume.

Spine density analysis. Following saline perfusion, mice were perfused with aldehyde fixative solution (Bioenno, \#003780). Brains were then carefully removed and placed in fixative solution at $4^{\circ} \mathrm{C}$ overnight. Brains were then sliced at $100-\mu \mathrm{m}$-thick vibratome sections and immersed in impregnation solution (Bioenno, sliceGolgi Kit, \#003760) for $5 \mathrm{~d}$. Further staining was performed as described by the manufacturer (Bioenno). Images of dendrites were obtained at the same anatomical level for all brains, $400 \mu \mathrm{m}$ from the lesion perimeter in cortical layer $2 / 3$. In total, 5 dendrites from 5 neurons each in both hemispheres were imaged $(100 \times$ brightfield). Dendrites from the images were then reconstructed using Imaris $\times 64$ (version 8.4.0, Bitplane).

Immunohistochemistry. Mice were perfused with saline and then $4 \%$ PFA. Brains were removed and postfixed in $4 \%$ PFA at $4^{\circ} \mathrm{C}$ overnight. Brains were then dehydrated in $30 \%$ sucrose in $1 \times$ PBS. Microglia staining was performed on $100-\mu \mathrm{m}$-thick sections using rabbit anti-ibal (1: 200, Wako, \#019-19741), and then goat anti-rabbit 594 (1:200, Thermo Fisher Scientific, \#A-11012). Automated analysis of microglial cell counts and morphology was performed using a MATLAB-automated morphology protocol as previously described (Heindl et al., 2018). For microglial costaining, we used anti-CD68 (1:300, Bio-Rad, \#MCA1957GA), anti-inducible nitric oxide synthases (iNOS) (1:300, Thermo Fisher Scientific, \#MA5-17139), and anti-Arginase1 (1:300, Novus Biologicals, \#NB100-59740SS), and the corresponding secondary antibodies. Coverage analysis was performed using ImageJ software. Quantification was done for overlapping activation markers with Iba- 1 from the maximum intensity projections generated from $z$ stacks of the ipsilateral cortex. For synaptic staining, sections were incubated with guinea pig anti-VGlut1 (1:1000, Millipore, \#AB5905) and chicken anti-Homer 1 (1:2000, Synaptic Systems, \#160006) for $3 \mathrm{~d}$ at $4^{\circ} \mathrm{C}$, and then counterstained with goat anti-guinea pig 488 (1:500, Invitrogen, \#A11073) and goat anti-chicken 647 (1:500, Invitrogen, \#A21449) for $18 \mathrm{~h}$ at room temperature. For synaptic puncta analysis, a $z$ stack of 3 slices at $0.33 \mu \mathrm{m}$ was used as previously described (Ippolito and Eroglu, 2010) using FIJI with the SynapseCounter plugin (Schindelin et al., 2012).

Flow cytometric analysis. Following saline perfusion in deep anesthesia, the entire spleen and both brain hemispheres were dissected. Spleens were immediately placed on ice-cold $1 \times$ PBS, passed through a $40 \mu \mathrm{m}$ cell strainer, and treated with red blood cell lysis buffer. Cell surface markers were stained using the following antibodies: anti-CD3 FITC (clone 17A2) and anti-CD45 eFluor450 (clone 30-F11). Stained cells were analyzed on a BD FACSVerse flow cytometer (BD Biosciences) and analysis performed by FlowJo software (version 10.0). For brain, hemispheres were isolated and cells isolated by mechanical dissociation. Mononuclear cells were enriched using discontinuous Percoll gradient centrifugation by standard protocols and then with $30 \mu \mathrm{m}$ cell strainer, as described previously (Benakis et al., 2016). Cell staining for flow cytometry was performed by first preincubation of cells with Fc receptor blocker (1:100, Invitrogen, 14-91-61-73) for $10 \mathrm{~min}$ and then staining with the following monoclonal antibodies: anti-CD3 FITC (clone 17A2), anti-CD45 eFluor450 (clone 30-F11), and anti-CD11b Pe-Cy7 (clone M1/70). Stained cells were analyzed on a BD FACSVerse flow cytometer (BD Biosciences) and analysis performed by FlowJo software (version 10.0).

RNA sequencing and data analysis. Fourteen days after stroke, brains were extracted from the skulls and placed into a stainless-steel brain matrix. Brains were sliced coronally at $2 \mathrm{~mm}$ distance caudally and rostrally away from the lesion. The cortex was removed, frozen on dry ice, and the mRNA isolated using the RNeasy Mini Kit (QIAGEN, \#74109). For library preparation, $100 \mathrm{ng}$ of total RNA was fragmented and processed using the Ovation Human FFPE RNA-Seq Library Systems (Nugen) according to the instructions of the manufacturer.

Barcoded libraries were quantified using the Library Quantification Kit-Illumina/Universal (KAPA Biosystems). Cluster generation was performed with a concentration of $10 \mathrm{~nm}$ using a cBot (Illumina). Sequencing of $2 \times 100 \mathrm{bp}$ paired-end reads was performed with a HiScanSQ sequencing platform (Illumina) using version 3 chemistry at the sequencing core facility of the IZKF Leipzig (Faculty of Medicine, University Leipzig, Leipzig, Germany). Raw reads were mapped to the reference genome mm10 using split-read mapping algorithm implemented in segemehl (Hoffmann et al., 2009). Mapped reads were counted using feature Counts (Liao et al., 2014) according to RefSeq annotation. Differential expression was computed using DESeq2 algorithm (Love et al., 2014). Raw data have been deposited at Gene Expression Omnibus database (Accession number GSE131788).

$R T-P C R$. mRNA from brain tissue around the lesion was isolated as described above and transcribed using the High Capacity cDNA Reverse Transcription Kit (Applied Biosystems, \#4368814) with Rnasin Plus RNase Inhibitor (Promega, \#N2611). The following primers were used: BDNF (forward: CGGCGCCCATGAAAGAAGTA; reverse: AGACCT CTCGAACCTGCCCT), TrkB (forward: ACTTCGCCAGCAGTAGCAG; reverse: ACCTCAGGGCTGGGGAG), synaptophysin (forward: AGTACCCATTCAGGCTGCAC; reverse: CCGAGGAGGAGTAGTCA CCA), EphrinA5 (forward: CTGGTGCTCTGGATGTGTGT; reverse: CCCTCTGGAATCTGGGGTTG), PPIA (forward: ACACGCCATAATG GCACTGG; reverse: ATTTGCCATGGACAAGATGCC), Claudin-5 (forward: GTTAAGGCACGGGTAGCACT; reverse: TACTTCTGTG ACACCGGCAC), ICAM-1 (forward: CAATTTCTCATGCCGCACAG; reverse: AGCTGGAAGATCGAAAGTCCG), and VCAM-1 (forward: TGAACCCAAACAGAGGCAGAG; reverse: GGTATCCCATCACTTG AGCAGG). The QuantiNova SYBR Green PCR Kit (QIAGEN, \#208052) was used with a LightCycler 480 II (Roche Diagnostics). All gene expression was expressed relative to the PPIA housekeeping gene and calculated using the relative standard curve method.

Experimental design and statistical analysis. All statistical analyses were performed using Prism software (GraphPad, version 6.0). Sample size was chosen based on comparable experiments from previous experiments (Singh et al., 2016). For experimental design details on sample size, see Results and figure legends. A $p$ value of $<0.05$ was regarded as statistically significant. 


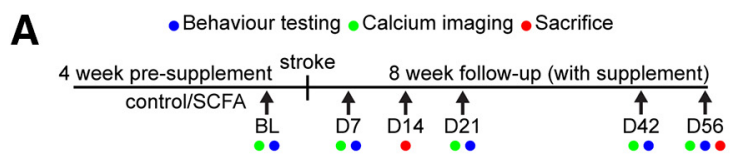

B
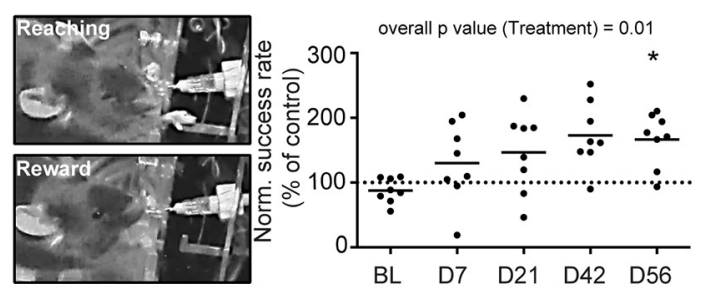

D

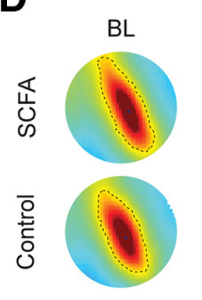

D21

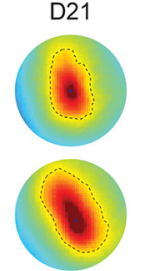

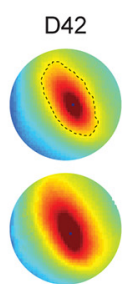

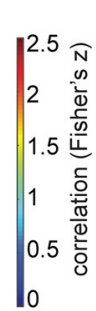

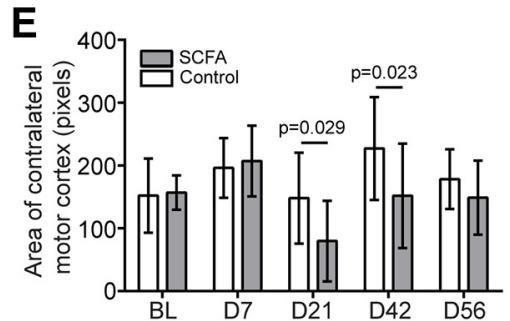

Figure 1. SCFA supplementation improves recovery after stroke. $A$, Schematic diagram illustrating the timeline of SCFA supplementation and analysis time points. BL, Baseline; D, day (after stroke). See Figure 1-1 (available at https://doi.org/10.1523/JNEUROSCI.1359-19.2019.f1-1). B, Representative images obtained during the lever pull test of trained mouse successfully reaching for the lever (left, above) and obtaining the peanut oil reward (left, below). Right, Normalized success rate for lever pulls by the affected (contralesional) forelimb. Relative values are shown per time point normalized to the mean of the control group. $N=8$ per group. Horizontal line indicates mean. Two-way repeated measure ANOVA with Holm-Sidak's post hoc test. C, Topographic depiction of seed-based functional connectivity of both hemispheres at indicated time points of SCFAs and control-treated mice. Seed is placed in the homotypic contralesional region to the ipsilesional lesion area (i.e., the contralesional motor cortex). Color code represents Fisher's z correlation between the seed and every other pixel in the cortex. $\boldsymbol{D}$, Enlarged images of the contralesional motor cortex (region homotypic to the infarct lesion). Area highlighted with dotted line represents the highly connected functional motor cortex area (pixels with Fisher's $z$ values $>2.25$ ). $\boldsymbol{E}$, Quantification of highly correlated (Fisher's $z>2.25$ ) area of the contralesional motor cortex in control (open bars) and SCFA-treated mice (gray bars). $N=15$ per group. Multiple $t$ tests per time point with Holm-Sidak's correction for multiple testing.

\section{Results}

\section{SCFA supplementation improves recovery and cortical reorganization after stroke}

We previously showed that stroke induces dysbiosis of the gut microbiome with the hallmark of reduced bacterial diversity (Singh et al., 2016). To test the impact of poststroke gut dysbiosis on the metabolic function of the microbiome, we performed targeted analysis of plasma samples for SCFA concentrations after fMCAo stroke and sham surgery in mice by mass spectrometry. This analysis revealed significantly reduced plasma SCFA concentrations $3 \mathrm{~d}$ after fMCAo stroke surgery compared with shamoperated mice (Fig. 1-1A, available at https://doi.org/10.1523/ JNEUROSCI.1359-19.2019.f1-1). Therefore, we hypothesized that supplementation of SCFA would increase circulating SCFA concentrations and potentially induce therapeutic effects within the chronic poststroke recovery period. To test this hypothesis, we supplemented mice for 4 weeks with drinking water containing either a mix of acetate, butyrate, and propionate (for details, see Materials and Methods) or control drinking water with matched sodium chloride concentration. SCFA supplementation did not affect body weight (Fig. 1-1B, available at https://doi.org/ 10.1523/JNEUROSCI.1359-19.2019.f1-1) or overt behavior of the animals. We performed PT stroke surgery after 4 weeks of SCFA supplementation and assessed poststroke motor deficits of the affected forelimb with an automated lever pull test while animals received further SCFA supplementation during the complete survival period (Fig. $1 A, B$ ). Mice receiving SCFA supplementation performed significantly better compared with control-treated animals (Fig. 1B). A two-way ANOVA for re- peated measurements revealed an overall $p$ value of 0.01 for the treatment effect, and a significant difference (after correction for multiple comparisons) between treatment groups at $56 \mathrm{~d}$ after stroke (Fig. $1 B$ ). To analyze cortical network plasticity as the morphological surrogate of behavioral recovery, we used Thy1GCaMP6s mice and performed resting-state in vivo calcium to record cortical wide-field fluorescence from a neuronal based calcium reporter. Using the homotypical contralesional region of the cortex, we performed seed-based correlation analysis, indicating the connectivity strength ( $z$ score) of this area to every other pixel in the cortex (Fig. 1C). Previous research using fMRI in stroke patients has indicated that the homotypical contralesional region receives less neuronal inhibition from the stroked hemisphere, leading to disinhibition of the contralesional hemisphere (Rehme and Grefkes, 2013). To analyze the size of highly connected homotypical contralesional regions (i.e., the contralesional motor cortex), we measured the area of pixels with a $z$ score $>2.25$ (Fig. 1D). We observed a significantly reduced area of the contralesional motor cortex in SCFA- compared with control-treated mice at D21 and D42 (Fig. 1E). In contrast, SCFA treatment did not significantly affect the primary infarct area by in vivo imaging (Fig. 1-1C, available at https://doi.org/10.1523/ JNEUROSCI.1359-19.2019.f1-1) and histological analyses with infarct volumetry in three different focal stroke models after stroke (Fig. 2-1A-E, available at https://doi.org/10.1523/JNEUROSCI. 1359-19.2019.f2-1). These results indicated that, while SCFA supplementation did not affect the initial lesion development, SCFAs improved behavioral stroke outcome and modulated cortical network plasticity at later stages after stroke. 
A

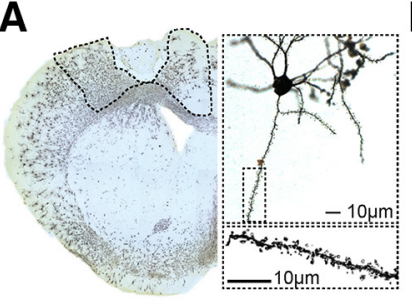

B

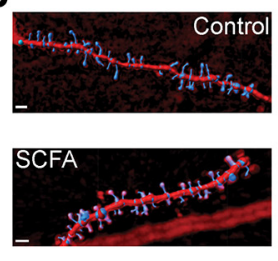

E

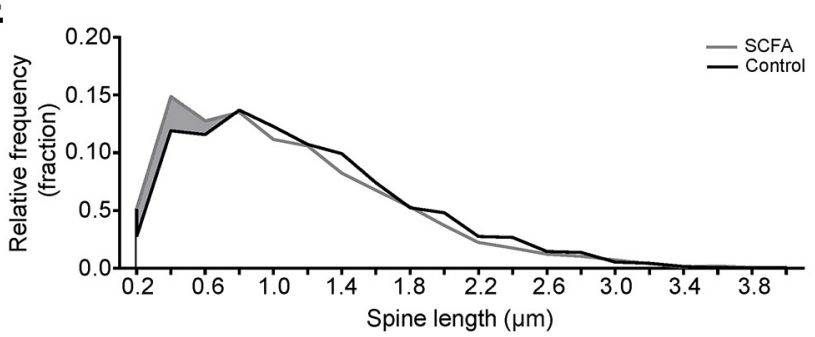

C

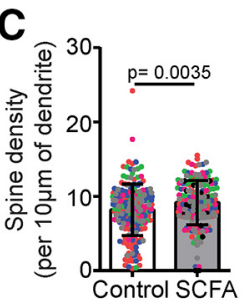

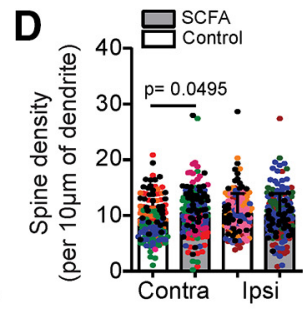

F
G
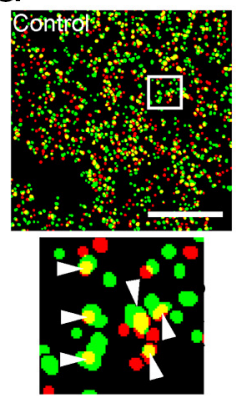

I

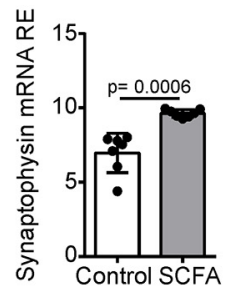

H
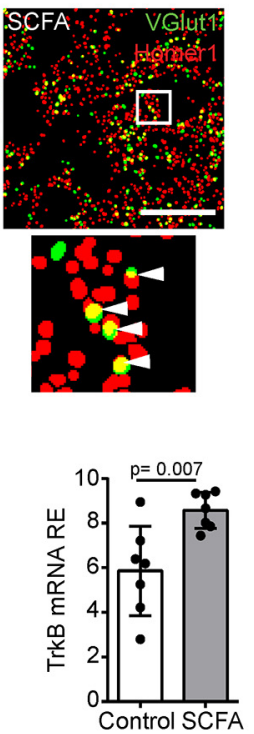
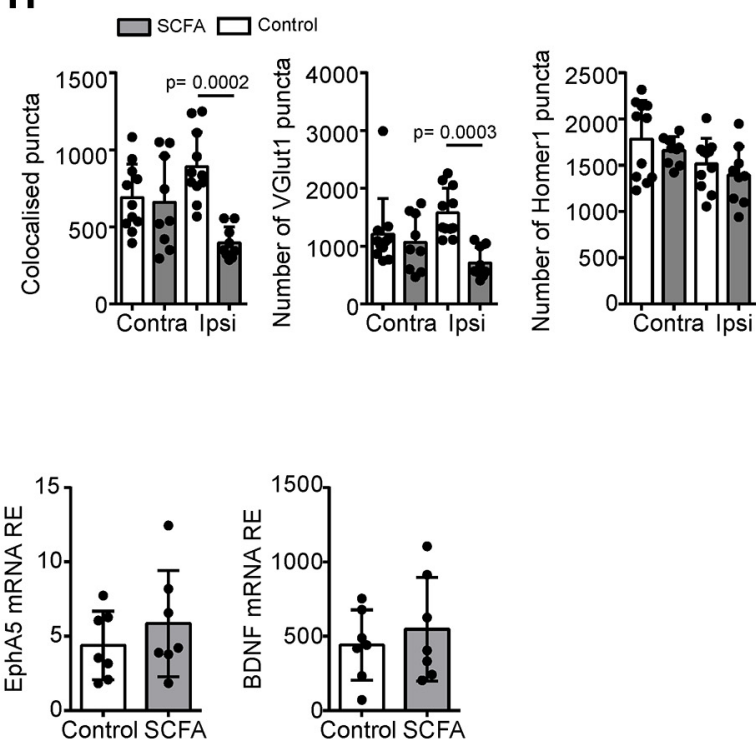

Figure 2. Poststroke neuronal plasticity is altered by SCFA treatment. $A$, Representative images of Golgi-Cox stained brain sections $14 \mathrm{~d}$ after PT stroke (Figure 2-1 $A-E$, available at https:// doi.org/10.1523/JNEUROSCI.1359-19.2019.f2-1). Dotted area in overview image represents perilesional cortical area used for spine analysis. Magnifications show representative pyramidal neuron and high-magnification image as used for spine analysis. Top right, Cortical pyramidal neuron. Bottom right, Example of spines identified on dendrite. Scale bar, $10 \mu \mathrm{m}$. B, 3D reconstruction for a dendrite section with spines as used for further quantification of spine densities and lengths. Scale bar, $2 \mu \mathrm{m}$. C, Quantification of pyramidal spine density per $10 \mu \mathrm{m}$ of dendrite in the cortex of control and SCFA-treated naive mice (no stroke induction). Each color represents a different mouse, and each dot indicates a different dendrite. Five neurons per hemisphere analyzed in total for 4 or 5 mice per group (Mann-Whitney U test). D, Quantification of spine density per $10 \mu \mathrm{m}$ of dendrite in the perilesional and contralesional cortex at $14 \mathrm{~d}$ after stroke (Kruskal-Wallis test with Dunn's correction for multiple comparisons). $\boldsymbol{E}$, Histogram of the relative frequency (fraction) of spines found at different lengths $14 \mathrm{~d}$ after PT lesion in the perilesional cortex. Bin width is $0.2 \mu \mathrm{m}$ (Figure 2-1F,G, available at https://doi.org/10.1523/JNEUROSCI.1359-19.2019.f2-1).F, Quantification of short (0.2 $\mu \mathrm{m}$ ) and long (1.4 $\mu \mathrm{m}$ ) spines in control (open bars) and SCFA (gray bars) treated mice. $N=4$ or 5 per group. Mann-Whitney $U$ test. G, Representative particle images of presynaptic terminals by VGlut1 (green), postsynaptic densities by Homer1 (red), and nuclei with DAPI (blue) of the cortex from control and SCFA-treated mice, as used for quantification of colocalized presynaptic and postsynaptic particles (puncta). Scale bar, $20 \mu \mathrm{m}$. Arrowheads indicate colocalized (yellow) puncta. $\boldsymbol{H}$, Synapse counts were quantified as colocalized VGlut1 and Homer1 puncta (Figure 2-1 H, available at https://doi.org/10.1523/JNEUROSCI.1359-19.2019.f2-1). Quantification for colocalization (left) and for single markers (middle and right) revealed significantly changed synapse counts as a result of the reduced number of VGlut $1^{+}$terminals in the perilesional cortex. contra, Contralesional hemisphere; ipsi, ipsilesional hemisphere. $N=3$ or 4 mice (3 sections per mouse). Statistical analysis was performed with Kruskal-Wallis test with Dunn's multiple comparison correction. $I$, Relative expression (RE) of mRNA for key molecules involved in synaptic plasticity (left), synaptophysin (left middle), TrkB (right middle), and EphA5 (right) BDNF from the perilesional cortex in control (open bars) and SCFA (gray bars) treated mice. $N=7$ per group. Mann-Whitney $U$ test.

\section{SCFA supplementation modulates poststroke synaptic plasticity}

After an ischemic brain injury, the entire cortex undergoes rapid functional and morphological reorganization, including neuronal dendritic plasticity, which allows adult neurons to form new connections. This process is correlated with improved functional connectivity after a cortical lesion (Jones and Schallert, 1994; Biernaskie et al., 2004). To analyze underlying morphological plasticity of the observed functional recovery in behavior and cortical connectivity, we performed Golgi-Cox staining of brain sections to investigate the effects of SCFA supplementation on dendritic spine density of pyramidal cells (Fig. $2 A, B$ ). We initially performed this analysis in the brains of naive animals that received either SCFA or control treatment, which revealed a higher pyramidal cortical spine density in the SCFA-treated mice (Fig. 2C). Next, we quantified brains at $14 \mathrm{~d}$ after PT stroke to capture a time period before the behavioral and cortical connectivity improvements were evident. Correspondingly, we observed 
A

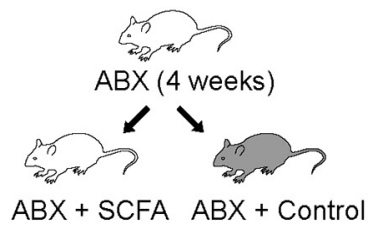

(4 weeks)

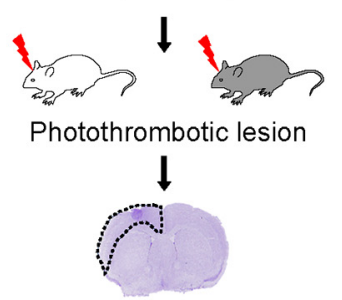

Isolation of ipsilateral cortex (post 2 weeks)
B

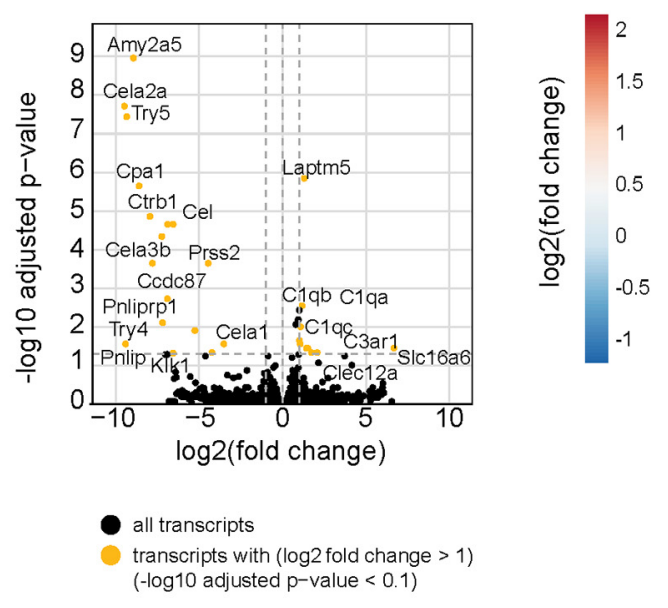

$E$

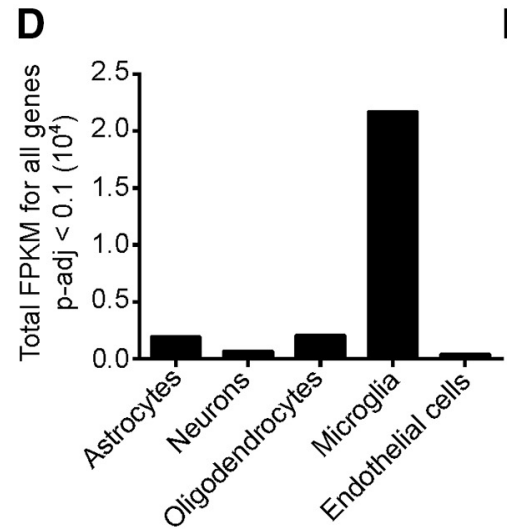

C

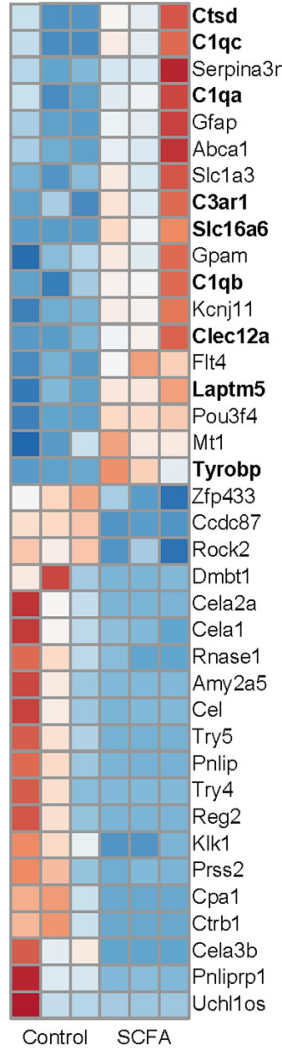

Figure 3. SCFA supplementation affects microglial gene signature after stroke. $A$, Schematic diagram illustrating antibiotic (ABX) treatment regimen and followed by supplementation with SCFAs or control saline in drinking water (Figure 3-1, available at https://doi.org/10.1523/JNEUROSCI.1359-19.2019.f3-1). Delineated area on histological image represents perilesional cortex isolated for mRNA sequencing. $\boldsymbol{B}$, Volcano plot of regulated transcripts (SCFAs/control) in the perilesional cortex $14 \mathrm{~d}$ after PT stroke; $n=3$ per group. Black represents gene transcripts. Orange represents gene transcripts with fold change $(\log 2)>1$ and $-\log 10$-adjusted $p<0.1$. C Heatmap of fold change $(\log 2)$ for significantly regulated genes with an adjusted $p<0.1$. Each column represents 1 individual mouse. $\boldsymbol{D}$, FPKN abundance and association per cerebral cell type of all significantly regulated genes ( $\boldsymbol{C}$ ) were performed as detailed in Materials and Methods, revealing the strongest association of the significantly regulated genes with microglial cells. $E$, Ingenuity pathway analysis showing the top networks regulated by SCFA supplementation compared with control treatment.

that, after stroke, SCFA supplementation was associated with a significantly higher spine density in the homotypic contralesional region; however, these differences were not observed in the ipsilesional hemisphere (Fig. 2D) and were independent of infarct volume (Fig. 2-1, available at https://doi.org/10.1523/JNEUROSCI. 1359-19.2019.f2-1). Moreover, the analysis of spine length distribution revealed that SCFA treatment induced a shift toward shorter spine lengths, specifically in the ipsilateral, but not contralesional, hemisphere (Fig. 2E, F; Fig. 2-1F, G, available at https://doi.org/10.1523/JNEUROSCI.1359-19.2019.f2-1). To further investigate synaptic plasticity under control of SCFA supplementation, we assessed synaptic density using costaining for VGlut1 (presynaptic) and Homer1 (postsynaptic) (Fig. 2G). We detected a significant reduction of the number of synapses (i.e., colocalized VGlut1 and Homer1 puncta) in the perilesional cortex of SCFA-supplemented mice (Fig. $2 H$ ). Interestingly, the difference in synapse counts was exclusively driven by the number of presynaptic VGlut1 puncta while Homer1-positive puncta remained unaffected.

Additionally, this pattern was also mirrored in the mean size of VGlut1 and Homer1 puncta (Fig. 2-1 H, available at https://
doi.org/10.1523/JNEUROSCI.1359-19.2019.f2-1). Finally, we analyzed the transcriptional regulation of key factors involved in synaptic plasticity (Fig. 2I). We observed that SCFAs significantly increased the expression of the presynaptic vesicle molecule synaptophysin and the BDNF receptor TrkB. BDNF itself, or the receptor tyrosine kinase EphA2, was not affected by SCFA supplementation. These results indicate effects of SCFA on morphological, synaptic plasticity, which could potentially precede the effects observed on functional recovery at later time points after stroke.

Brain transcriptomic analysis indicates microglia as the main cellular target of SCFA

To determine whether the observed effects of SCFA were either directly mediated on neuronal function or affecting other cerebral cell populations, we took an unbiased approach to investigate the effects of SCFA on gene expression in the peri-infarct cortex. For this, we first depleted the gut microbiome of mice as the main SCFA source by administration of antibiotics, followed by supplementation of SCFA (Fig. 3A). Plasma gas chromatographymass spectrometry quantification confirmed an increase of SCFA 
after supplementation in drinking water (Fig. 3-1, available at https://doi.org/10.1523/JNEUROSCI.1359-19.2019.f3-1). Fourteen days after surgery, the infarct and peri-infarct regions were isolated for RNA sequencing, identifying 18 upregulated and 20 downregulated genes in SCFA supplemented mice (Fig. $3 B, C$ ).

The list of the top upregulated genes (Fig. $3 C$ ) hinted at a role for microglia as we found numerous genes that have previously been reported to be involved in microglial function and/or activation, such as Ctsd, various complement molecules, Tyrobp and Laptm5 (Zhong et al., 2018; Q. Li et al., 2019). To ascertain which cell type SCFA supplementation was mainly regulating in the brain, we took the 38 differentially regulated genes and compared them with an existing RNA-Seq database, which lists the fragments per kilobase of exon model per million reads found in astrocytes, neurons, oligodendrocytes, microglia, and endothelial cells (www.brainrnaseq.org) (Zhang et al., 2014). From the total number of fragments per kilobase of exon model per million read values of the 38 differentially regulated genes (Table 1-1, available at https:// doi.org/10.1523/JNEUROSCI.1359-19.2019.t1-1), we discovered that the vast majority of gene reads from the significantly regulated genes were associated with microglial cells (Fig. 3D). Additionally, we performed an ingenuity pathway analysis on the 38 differentially regulated genes and found that the complement system was the top pathway and highly upregulated in mice supplemented with SCFA (Fig. $3 E$ ). Within the brain, the complement pathway is critical for microglia activation and has been associated with synaptic pruning by microglia (Schafer et al., 2012; Stephan et al., 2012; Wu et al., 2015).

\section{SCFAs modulate microglial activation and immune cell composition}

Based on the transcriptomic data's indication that microglia are the effector of SCFA-mediated poststroke recovery, we performed more in-depth analyses of microglial activation and the inflammatory response to stroke. As a surrogate of microglia activation, we performed Iba-1 immunohistochemistry of the cortex (Fig. 4A) and assessed microglia morphology using automated analysis (Heindl et al., 2018). Fourteen days after stroke, cortical microglia from SCFA-supplemented mice displayed a significantly more ramified and less spherical (i.e., less activated) morphology compared with controls (Fig. 4B). Additionally, the total number of microglia were significantly reduced in SCFA- compared with control-treated mice (Fig. $4 \mathrm{C}$ ). These results indicate a modulation of the microglial response to stroke by SCFAs. Therefore, we further explored microglial function by assessing classical histological markers for microglial activation and polarization. We observed a significant reduction of CD68 expression in Iba- ${ }^{+}$ microglia (Fig. 4D), whereas Arginase-1 and iNOS were not significantly regulated by SCFA supplementation (Fig. 4E). Aside from evoking a microglial response, stroke induces invasion of peripheral immune cells into the brain parenchyma, which can deteriorate stroke outcome (Iadecola and Anrather, 2011; Liesz et al., 2015; Neumann et al., 2015; Selvaraj and Stowe, 2017). In particular, lymphocyte counts in brains after photothrombosis are still elevated $14 \mathrm{~d}$ after surgery (Feng et al., 2017). Additionally, cytokines secreted by brain-invading lymphocytes can modulate microglial activation (Liesz et al., 2009; Meng et al., 2019). Therefore, we performed flow cytometry of brain homogenates (Fig. 4F) and observed a significant reduction in cerebral lymphocyte invasion in SCFA-treated animals compared with controls (Fig. 4G).

The invasion of peripheral lymphocytes to the ischemic brain depends on the number of circulating lymphocytes, the expres- sion of cerebral endothelial adhesion molecules, and the chemokine gradient derived from the injured brain tissue (Ransohoff and Engelhardt, 2012). In accordance with our nontargeted transcriptomic analysis (for results, see Fig. 3), which did not reveal significant regulation of chemokines or adhesion molecules by SCFAs, also a targeted PCR analysis of key adhesion molecules and tight junction proteins involved in poststroke lymphocyte recruitment at the blood-brain barrier did not show a significant regulation by SCFA (Fig. 4-1, available at https://doi.org/10.1523/ JNEUROSCI.1359-19.2019.f4-1). In contrast, we detected a significant reduction of systemic T-cell counts by SCFA supplementation in the spleen (Fig. 4H), a secondary lymphatic organ that well characterizes the systemic immune response after stroke (Offner et al., 2006; Liesz et al., 2013; Roth et al., 2018).

These findings suggest that SCFAs may primarily affect lymphocytes already in the peripheral immune compartments, which then might secondarily mediate changes in the cerebral immune milieu after brain invasion. Therefore, we next aimed to test this hypothesis by investigating the effects of SCFA on microglia in the absence of lymphocytes. For this, we used lymphocyte-deficient Rag1 ${ }^{-1-}$ mice, which were given SCFA supplementation or control drinking water. In contrast to lymphocyte-competent WT mice, SCFA supplementation in $R a g 1^{-I-}$ did neither affect microglia morphology, nor did it reduce microglial cell counts (Fig. $4 I, J)$, indicating a key role of lymphocytes for mediating the SCFA effects on microglia.

\section{Discussion}

This study demonstrates that SCFAs, critical metabolites derived from the gut microbiome, are capable of improving poststroke recovery via the modulating effects of brain-invading lymphocytes on microglial function. Results from our study support this conclusion through several key findings. First, stroke lowers blood SCFA concentrations. Second, SCFA supplementation combats the deleterious effects of this poststroke response, which we demonstrated by associated SCFA supplementation with improved behavioral recovery, changes in cortical network connectivity, which are generally associated with improved stroke outcomes, and changes in histological markers of synaptic plasticity. Third, we indicated a potential mechanism for the observed improvements in the recovery of SCFA-treated animals by finding changes in microglial function, which were dependent on circulating lymphocytes. Consequently, these findings indicate that SCFAs affect peripheral lymphocytes, maturation, or egress from primary lymphatic tissue, and lymphocytes then indirectly mediate the SCFA effects on the brain micromilieu either by their overall reduction in cerebral invasion or polarization of the secreted cytokine profile.

These novel findings introduce SCFA as the most likely missing link in the pathophysiological function of the gut-brain axis in stroke and poststroke recovery, for which the microbiotaderived effector molecules were so far unknown (Benakis et al., 2016; Houlden et al., 2016; Singh et al., 2016). Our use of a novel imaging modality, in vivo wide-field calcium imaging, provided a highly sensitive tool for assessing cortical network plasticity after stroke (Cramer et al., 2019). This tool allowed us to perform analyses of network plasticity by comparable analytical approaches to fMRI in patients, with the exception of using a genetically encoded reporter for direct neuronal activation instead of the blood flow surrogate marker (BOLD) used in MRI (Cramer et al., 2019). The analysis of connectivity within the cortical network provides information about the dynamic changes in defined cortical areas under resting-state conditions, which gave us 
A Iba-1 DAPI

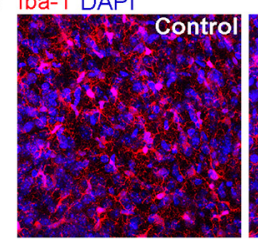

D

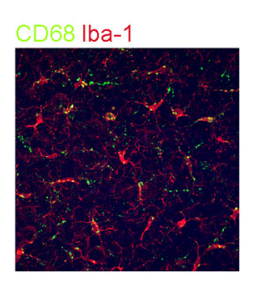

F
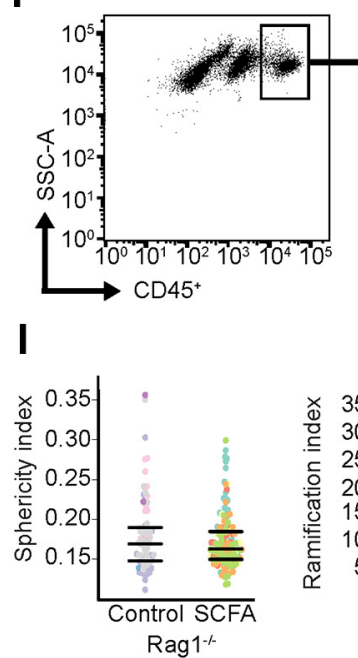

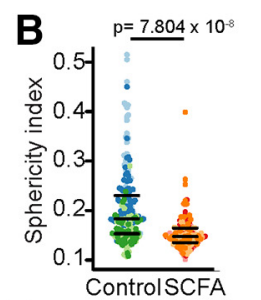

E

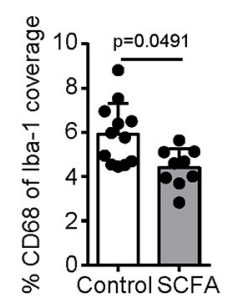

SCFA
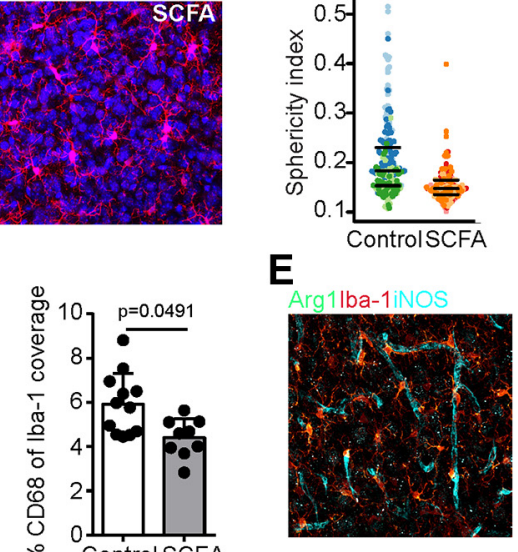
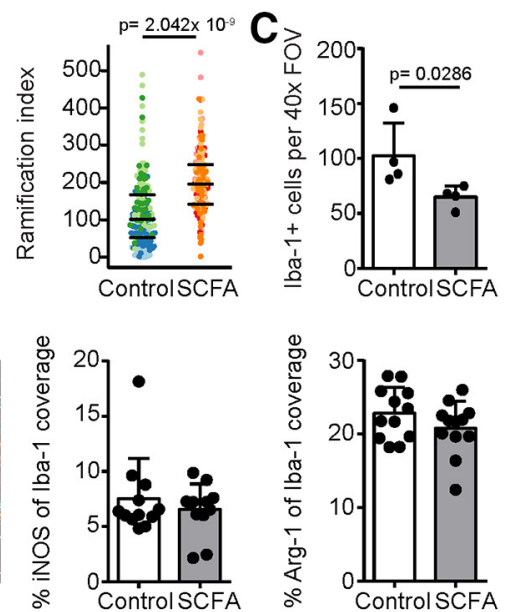

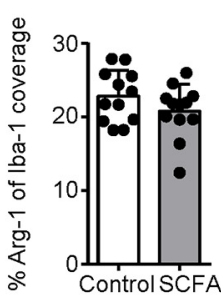

H
G

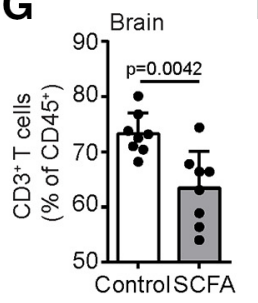

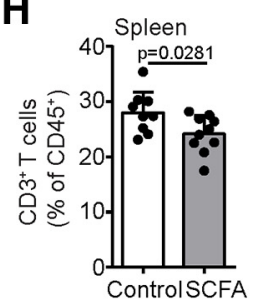

J
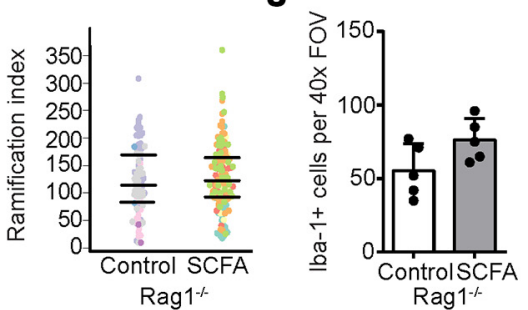

Figure 4. Modulation of poststroke neuroinflammation by SCFA depends on peripheral lymphocytes. $A$, Representative maximum intensity projections of microglial staining using Iba-1 (red) and DAPI (blue) in the ipsilesional hemisphere $14 \mathrm{~d}$ after PT stroke with either control (left) or SCFA (right) supplementation. B, Microglial morphology was analyzed in 3D using an automated analysis algorithm in the ipsilesional cortex of mice $14 \mathrm{~d}$ after stroke, which revealed significantly reduced sphericity (left) and increased number of branch nodes (right) as markers of reduced microglial activation by SCFA compared with control treatment. Each symbol represents one microglia. Different colors group together microglia from the same mouse. $C$, Number of microglia found per 1 high-power (40X) FOV in the perilesional cortex. D, Coexpression coverage analysis in the ipsilateral hemispheric cortex for CD68 and Iba- 1 expressed as percentage of Iba- 1 from a maximum intensity projection. Representative immunofluorescence image (red represents microglia; green represents (D68) (left) and quantification (right). $\boldsymbol{E}$, Coexpression coverage analysis in the ipsilateral hemispheric cortex for iNOS and Arginase 1 (Arg1) with Iba-1 expressed as percentage of lba-1 coverage area from a maximum intensity projection. Representative immunofluorescence image (red represents microglia; green represents Arg1; cyan represents iNOS) (left) and quantification (right). $N=3$ mice per group and 3 images per hemisphere. In contrast to the effects of $S C F A$ on microglia function, endothelial cells were unaffected by the SCFA treatment (Figure 4-1, available at https://doi.org/10.1523/JNEUROSCI.1359-19.2019.f4-1). $\boldsymbol{F}$, Representative gating strategy for flow cytometric analysis of T cells $\left(\mathrm{CD} 45^{+} \mathrm{CD}^{+}\right)$. SCFA supplementation significantly decreased the frequency of $\mathrm{T}$ cells in $(\boldsymbol{G})$ brains and $(\boldsymbol{H})$ spleens $14 \mathrm{~d}$ after stroke. $\mathrm{N}=9$ per group. Quantification of $(I)$ sphericity (left) and ramification index (right) and $(J)$ absolute cell counts of microglia $14 \mathrm{~d}$ after stroke in the perilesional cortex of Rag $1^{-/-}$mice. In contrast to WT mice (compare with $B, C$ ) SCFA treatment did not affect microglia activation in lymphocyte-deficient $R a g 1^{-1-}$ mice. All statistical analyses in this figure were performed using the Mann-Whitney $U$ test.

a unique ability to measure even the more subtle effects of SCFA supplementation on poststroke plasticity.

In human stroke patients, interhemispheric resting-state connectivity is significantly weakened following stroke. Specifically, it is thought that inhibitory projections from the lesioned hemisphere to the homotypic contralesional hemisphere are attenuated, leading to a disinhibition of the homotypic area in the contralesional hemisphere (Rehme and Grefkes, 2013). We confirmed this effect with our optogenetic imaging approach, by observing an increase in the contralesional motor cortex area as an indicator of reduced transhemispheric inhibition. This "blooming" effect of the contralesional motor cortex was significantly ameliorated by the SCFA treatment at chronic time points after stroke, which could indicate an improvement of interhemi- spheric connectivity and thereby reestablish inhibition of the contralesional hemisphere. However, the further in-depth exploration of such interhemispheric inhibition is impeded by several technical limitations of our imaging tool, such as autofluorescent artifacts in the perilesional territory and the lack of directional information of the interhemispheric connections. This would require in vivo electrophysiological studies on connectivity between the recovering perilesional tissue and the homotypic contralesional brain area, which are currently not yet established in stroke research. The functional relevance for the changes within connectivity was ultimately confirmed by the corresponding beneficial effects of SCFAs on recovery of motor functions. Behavioral recovery was assessed in a highly sensitive, rater-blinded, and high-throughput automated test, specifically analyzing mo- 
tor deficits of the affected forelimb, hence providing a reliable readout for poststroke recovery, even in the chronic phase, something that most conventional behavior tests lose their test sensitivity toward (Zausinger et al., 2000; X. Li et al., 2004; Manwani et al., 2011; Rosell et al., 2013).

As an independent line of evidence for the effects of SCFA on poststroke recovery, we detected significant changes in dendritic spine densities and synaptic counts based on histological analysis. Previous studies have demonstrated changes in dendritic spine density of the perilesional cortex as well as contralateral hemisphere and have been identified as a hallmark of poststroke tissue remodeling and marker of synaptic plasticity (Brown et al., 2008; Huang et al., 2018). After stroke, it has been shown that there is a gradual increase of spine density in the contralateral cortex (Huang et al., 2018), whereas spine density in the peri-infarct region is reduced by $\sim 38 \%$ within the acute phase (Brown et al., 2008). Accordingly, we observed significantly increased spine densities in the contralateral motor cortex of SCFA-supplemented mice, suggesting that these microbial metabolites could aid recovery by promoting dendritic spine plasticity.

A key limitation of the various analysis readouts used in this study (calcium imaging, spine density, synapse count) is their exclusive reflection of processes in excitatory neurons. The expression of GCamp in Thyl-positive neurons, analysis of dendritic spines on pyramidal neurons, and the synapse count of VGlut1-positive (glutamatergic) synapses all limit the analysis to the excitatory system of the forebrain cortex and do not account for potential alterations in subcortical structures or the complex integration of information by (inhibitory) interneurons within the cortex (Markram et al., 2004). However, the focus on the excitatory cortical system across the different methodological approaches allowed us to thoroughly investigate the impact of SCFAs from a global network perspective (wide-field imaging) to synaptic (sub)structures within the same system. This approach revealed a striking alignment of results across different functional levels from synapse to network plasticity and behavior that strongly support the impact of SCFAs on poststroke recovery. Despite this, future studies need to address the intricate regulation of cortical microcircuits underlying this effect and particularly the role of the various interneuron types. Another relevant limitation is the use only of male mice for most of the experiments (except dendritic spine analysis). Considering recent evidence for sexspecific effects along the gut-brain axis and particularly on microglial activation (Thion et al., 2018; Villa et al., 2018), we cannot exclude a differential effect of the observed pathway in the female sex.

The transcriptomic analysis performed in this study indicated several genes which have previously been associated with microglial activity and phagocytic function to be regulated by SCFAs in the poststroke recovery phase. In contrast, only a very low amount of significantly regulated genes by the SCFA supplementation were enriched for other cell population marker genes, such as neurons or endothelial cells. Therefore, we hypothesized that the SCFA effect on poststroke recovery and neuronal plasticity might be indirectly mediated via microglial activity. Indeed, microglia are key players involved in synaptic pruning and dendritic remodeling (Salter and Stevens, 2017). Upon proinflammatory stimuli, microglial activation is associated with a change in cell morphology. While homeostatic microglia are highly ramified, activated cells acquire a more ameboid shape. We observed after stroke an increase in cell ramification by the SCFA supplementation, indicating a reduced microglial activation state. Interestingly, microglial activation after stroke has previously been associated with reduced phagocytic capacities (Faustino et al., 2011). Hence, modification of microglial activation by SCFA could have an impact on synapse elimination and thereby microglia-dependent synaptic plasticity (Salter and Stevens, 2017). However, microglial elimination ("pruning") of dendritic synapses is vice versa associated with synaptic activity (i.e., less active presynaptic inputs are more likely to be phagocytosed). Reduced synaptic activity in the perilesional cortex due to the tissue injury or in the contralesional cortex due to loss of transcallosal innervation from the injured cortex could both induce excessive (pathological) dendrite pruning (Brown et al., 2008; Riccomagno and Kolodkin, 2015; Salter and Stevens, 2017). This would suggest that dendritic spine remodeling and microglial activation are a reciprocal, bidirectional interaction of neurons and microglia in the recovering cortex after stroke. While the results of RNASeq analysis indicate that SCFAs modulate primarily microglial activation in this process, the detailed interaction of neurons and microglia as well as the directionality of their association will require further experimental studies. Finally, microglia activation has been associated with neuronal plasticity and function beyond morphological reorganization and spine remodeling. We have recently demonstrated that microglia monitor neuronal function at the cell soma and this somatic interaction are altered during pathology after stroke (Cserép et al., 2019), a process that could likely contribute to the functional changes observed in cortical calcium imaging and behavioral outcomes. However, the contribution of SCFAs to this somatic cell-cell interaction is so far unknown.

A large part of previous experimental studies with SCFA have focused on their effect on circulating immune cells. SCFAs modulate the polarization of lymphocyte subsets, such as the ratio of anti-inflammatory regulatory $\mathrm{T}$ cells (Treg) to proinflammatory $\mathrm{T}_{\mathrm{H}} 17$ cells (Arpaia et al., 2013; Haghikia et al., 2015; Park et al., 2015; Asarat et al., 2016), as well as their migratory behavior between organs (Nakamura et al., 2017). Importantly, lymphocyte invasion to the ischemic brain is a hallmark of poststroke neuroinflammation (Iadecola and Anrather, 2011). It is therefore likely that SCFAs may not exclusively act directly on resident microglia per se, but also via the peripheral effects of SCFAs, which change lymphocyte function. The altered peripheral lymphocytes later invade the injured brain and indirectly influence the cerebral inflammatory milieu. Indeed, T cells have been consistently identified in several reports as the invading leukocyte subpopulation with the largest impact on stroke outcome with partially divergent functions of T-cell subsets (Kleinschnitz et al., 2010). On one hand, immunosuppressive Treg cells provide a neuroprotective function by suppressing an overshooting inflammatory reaction to the brain infarct (Liesz et al., 2015); on the other hand, the proinflammatory $\mathrm{T}_{\mathrm{H}} 1$ and $\mathrm{T}_{\mathrm{H}} 17$ cells induce secondary neurotoxicity and lead to infarct expansion with worsened functional outcome (Shichita et al., 2009; Gelderblom et al., 2012). In addition to the impact of SCFAs on T-cell polarization, alternative mechanisms of immunoactive SCFA effects might be mediated via regulation of micro-RNA expression (so far mainly studied in cancer) or by modulating the production of reactive oxygen species; however, these pathways are still under investigation and require further exploration in stroke.

Several experimental stroke models have been established for modeling focal ischemic brain injury in rodents. While some stroke models use occlusion of a large cerebral artery, such as intraluminal occlusion of the MCA by a suture or the direct, distal MCA occlusion model, other stroke models induce artery occlusion using other strategies, such as end arterial occlusion by photosensitive dyes or vasoconstriction using endothelin-1 (Fluri et 
al., 2015; Kumar et al., 2016). All of these experimental stroke methods only model certain elements of the highly complex pathophysiological events of stroke in human patients. Yet, each model provides the advantage to be particularly useful in analyzing specific targets of stroke interventions, acute neuroprotection, reperfusion, delayed apoptosis, inflammation, or chronic repair mechanisms (Carmichael, 2005). The PT stroke model, which was the main animal model used for most experiments in this study, was previously suggested as particularly suitable for analyzing recovery mechanisms after stroke, and is still widely used in this research domain (Carmichael, 2005; Murphy and Corbett, 2009). Disadvantages of this model are that it does not allow local collateral reflow and shows a profound local edema during the acute phase. Therefore, this model is not useful for studies to investigate acute neuroprotective agents or reperfusion therapies. On the other hand, PT stroke allows precise positioning of the lesion in functionally defined brain areas (i.e., the motor cortex in our study). Moreover, the highly reproducible and confined cortical lesion allows for a more detailed analysis of pathophysiological processes in the perilesional cortex compared with other stroke models; which are less consistent in the lesion localization.

Surprisingly, despite this obviously pronounced effect of lymphocytes in poststroke pathology, their role in activating or inhibiting microglial activation has so far barely been investigated. We have observed indirect evidence for T-cell effects on microglial activity in previous experiments, in which depletion of circulating Treg cells affected microglial activation and cytokine release (Liesz et al., 2009). Additional indirect evidence comes from studies demonstrating T-cell interaction and particularly the influence of $\mathrm{T}$ cell-secreted cytokines on multiple other cell types in the healthy and injured brain, such as T cell-derived interferon-gamma on animal behavior (Walsh et al., 2015; Filiano et al., 2016). Yet, to our knowledge, the role of T cells as indirect cellular mediators of microbiota-derived SCFAs on the brain has so far not been studied. In this study, we unequivocally demonstrate, for the first time, that $\mathrm{T}$ cells are crucial to mediate the immunomodulatory effects of SCFA on brain resident microglia because, in lymphocyte-deficient mice, this effect became abolished. This finding is also in accordance with our previous proof-of-concept experiments showing that circulating $\mathrm{T}$ cells were key in facilitating the impact of the gut microbiome on stroke outcome in germ-free versus recolonized animals as well as in mice with a healthy versus a dysbiotic microbiome (Singh et al., 2016, 2018). These previous reports by us and independent reports by others have also consistently identified a translocation of lymphocytes from the intestinal immune compartment to the poststroke brain, providing a cellular link by these circulating and highly motile cells across the gut-brain axis (Benakis et al., 2016; Singh et al., 2016).

Together, this study identified SCFAs as critical metabolites derived from the gut microbiome affecting $\mathrm{T}$-cell function and thereby indirectly modulating the neuro-regenerative milieu. This expands our current understanding of the mechanisms along the gut-brain axis in acute brain injury and recovery where poststroke dysbiosis affects the production of key microbiotaderived metabolites, their impact on immunological homeostasis, and finally the capability for efficient functional recovery. The efficacy of SCFAs for promoting recovery in an experimental stroke model on a functional as well as morphological level opens up novel therapeutic possibilities for improving recovery of human stroke patients. Future studies should validate the proregenerative effect of SCFAs on poststroke recovery before further translational development. Based on the findings from this study and indications for efficacy and similar modes of actions in primary autoimmune brain disorders (Melbye et al., 2019), it is well conceivable that SCFA supplementation could be used as a safe and practical add-on therapy to stroke rehabilitation. However, further studies will be required to test the efficacy of this approach in a post-treatment paradigm, in combination with common comorbidities and comedications, and finally validate in a confirmatory, multicenter preclinical study design before further translation to human stroke patients can be considered (Llovera et al., 2015; Llovera and Liesz, 2016).

\section{References}

Arpaia N, Campbell C, Fan X, Dikiy S, van der Veeken J, deRoos P, Liu H, Cross JR, Pfeffer K, Coffer PJ, Rudensky AY (2013) Metabolites produced by commensal bacteria promote peripheral regulatory T-cell generation. Nature 504:451-455.

Asarat M, Apostolopoulos V, Vasiljevic T, Donkor O (2016) Short-chain fatty acids regulate cytokines and Th17/Treg cells in human peripheral blood mononuclear cells in vitro. Immunol Invest 45:205-222.

Austin V, Ku JM, Miller AA, Vlahos R (2019) Ischaemic stroke in mice induces lung inflammation but not acute lung injury. Sci Rep 9:3622.

Becker AM, Meyers E, Sloan A, Rennaker R, Kilgard M, Goldberg MP (2016) An automated task for the training and assessment of distal forelimb function in a mouse model of ischemic stroke. J Neurosci Methods 258: $16-23$.

Benakis C, Brea D, Caballero S, Faraco G, Moore J, Murphy M, Sita G, Racchumi G, Ling L, Pamer EG, Iadecola C, Anrather J (2016) Commensal microbiota affects ischemic stroke outcome by regulating intestinal gamma delta T cells. Nat Med 22:516-523.

Bieber M, Werner RA, Tanai E, Hofmann U, Higuchi T, Schuh K, Heuschmann PU, Frantz S, Ritter O, Kraft P, Kleinschnitz C (2017) Strokeinduced chronic systolic dysfunction driven by sympathetic overactivity. Ann Neurol 82:729-743.

Biernaskie J, Chernenko G, Corbett D (2004) Efficacy of rehabilitative experience declines with time after focal ischemic brain injury. J Neurosci 24:1245-1254.

Bourassa MW, Alim I, Bultman SJ, Ratan RR (2016) Butyrate, neuroepigenetics and the gut microbiome: can a high fiber diet improve brain health? Neurosci Lett 625:56-63.

Brown CE, Wong C, Murphy TH (2008) Rapid morphologic plasticity of peri-infarct dendritic spines after focal ischemic stroke. Stroke 39:12861291.

Carmichael ST (2005) Rodent models of focal stroke: size, mechanism, and purpose. NeuroRx 2:396-409.

Chuang DM, Leng Y, Marinova Z, Kim HJ, Chiu CT (2009) Multiple roles of HDAC inhibition in neurodegenerative conditions. Trends Neurosci 32:591-601.

Cramer JV, Gesierich B, Roth S, Dichgans M, Düring M, Liesz A (2019) In vivo wide-field calcium imaging of the mouse cortex for analysis of network connectivity in health and brain disease. Neuroimage 199:570-584.

Cryan JF, Dinan TG (2012) Mind-altering microorganisms: the impact of the gut microbiota on brain and behaviour. Nat Rev Neurosci 13:701712 .

Cserép C, Pósfai B, Lénárt N, Fekete R, László ZI, Lele Z, Orsolits B, Molnár G, Heindl S, Schwarcz AD, Ujvári K, Környei Z, Tóth K, Szabadits E, Sperlágh B, Baranyi M, Csiba L, Hortobágyi T, Maglóczky Z, Martinecz B, et al. (2019) Microglia monitor and protect neuronal function via specialized somatic purinergic junctions. Science. Advance online publication. Retrieved December 12, 2019. doi: 10.1126/science.aax6752.

Dirnagl U, Iadecola C, Moskowitz MA (1999) Pathobiology of ischaemic stroke: an integrated view. Trends Neurosci 22:391-397.

Erny D, Hrabe de Angelis AL, Jaitin D, Wieghofer P, Staszewski O, David E, Keren-Shaul H, Mahlakoiv T, Jakobshagen K, Buch T, Schwierzeck V, Utermöhlen O, Chun E, Garrett WS, McCoy KD, Diefenbach A, Staeheli P, Stecher B, Amit I, Prinz M (2015) Host microbiota constantly control maturation and function of microglia in the CNS. Nat Neurosci 18:965977.

Faustino JV, Wang X, Johnson CE, Klibanov A, Derugin N, Wendland MF, Vexler ZS (2011) Microglial cells contribute to endogenous brain defenses after acute neonatal focal stroke. J Neurosci 31:12992-13001. 
Feng Y, Liao S, Wei C, Jia D, Wood K, Liu Q, Wang X, Shi FD, Jin WN (2017) Infiltration and persistence of lymphocytes during late-stage cerebral ischemia in middle cerebral artery occlusion and photothrombotic stroke models. J Neuroinflammation 14:248.

Filiano AJ, Xu Y, Tustison NJ, Marsh RL, Baker W, Smirnov I, Overall CC, Gadani SP, Turner SD, Weng Z, Peerzade SN, Chen H, Lee KS, Scott MM, Beenhakker MP, Litvak V, Kipnis J (2016) Unexpected role of interferongamma in regulating neuronal connectivity and social behaviour. Nature 535:425-429.

Fluri F, Schuhmann MK, Kleinschnitz C (2015) Animal models of ischemic stroke and their application in clinical research. Drug Des Dev Ther 9:3445-3454.

Frost G, Sleeth ML, Sahuri-Arisoylu M, Lizarbe B, Cerdan S, Brody L, Anastasovska J, Ghourab S, Hankir M, Zhang S, Carling D, Swann JR, Gibson G, Viardot A, Morrison D, Thomas EL, Bell JD (2014) The short-chain fatty acid acetate reduces appetite via a central homeostatic mechanism. Nat Commun 5:3611.

Gelderblom M, Weymar A, Bernreuther C, Velden J, Arunachalam P, Steinbach K, Orthey E, Arumugam TV, Leypoldt F, Simova O, Thom V, Friese MA, Prinz I, Hölscher C, Glatzel M, Korn T, Gerloff C, Tolosa E, Magnus T (2012) Neutralization of the IL-17 axis diminishes neutrophil invasion and protects from ischemic stroke. Blood 120:3793-3802.

Haghikia A, Jörg S, Duscha A, Berg J, Manzel A, Waschbisch A, Hammer A, Lee DH, May C, Wilck N, Balogh A, Ostermann AI, Schebb NH, Akkad DA, Grohme DA, Kleinewietfeld M, Kempa S, Thöne J, Demir S, Müller DN, et al. (2015) Dietary fatty acids directly impact central nervous system autoimmunity via the small intestine. Immunity 43:817-829.

Heindl S, Gesierich B, Benakis C, Llovera G, Duering M, Liesz A (2018) Automated morphological analysis of microglia after stroke. Front Cell Neurosci 12:106.

Hoffmann S, Otto C, Kurtz S, Sharma CM, Khaitovich P, Vogel J, Stadler PF, Hackermüller J (2009) Fast mapping of short sequences with mismatches, insertions and deletions using index structures. PLoS Comput Biol 5:e1000502.

Houlden A, Goldrick M, Brough D, Vizi ES, Lénárt N, Martinecz B, Roberts IS, Denes A (2016) Brain injury induces specific changes in the caecal microbiota of mice via altered autonomic activity and mucoprotein production. Brain Behav Immun 57:10-20.

Hoving LR, Heijink M, van Harmelen V, van Dijk KW, Giera M (2018) GC-MS analysis of short-chain fatty acids in feces, cecum content, and blood samples. Methods Mol Biol 1730:247-256.

Huang SY, Chang CH, Hung HY, Lin YW, Lee EJ (2018) Neuroanatomical and electrophysiological recovery in the contralateral intact cortex following transient focal cerebral ischemia in rats. Neurol Res 40:130-138.

Iadecola C, Anrather J (2011) The immunology of stroke: from mechanisms to translation. Nat Med 17:796-808.

Ippolito DM, Eroglu C (2010) Quantifying synapses: an immunocytochemistrybased assay to quantify synapse number. J Vis Exp 45:2270.

Jones TA, Schallert T (1994) Use-dependent growth of pyramidal neurons after neocortical damage. J Neurosci 14:2140-2152.

Kau AL, Ahern PP, Griffin NW, Goodman AL, Gordon JI (2011) Human nutrition, the gut microbiome and the immune system. Nature 474:327336.

Kim HJ, Leeds P, Chuang DM (2009) The HDAC inhibitor, sodium butyrate, stimulates neurogenesis in the ischemic brain. J Neurochem 110: $1226-1240$.

Kleinschnitz C, Schwab N, Kraft P, Hagedorn I, Dreykluft A, Schwarz T, Austinat M, Nieswandt B, Wiendl H, Stoll G (2010) Early detrimental T-cell effects in experimental cerebral ischemia are neither related to adaptive immunity nor thrombus formation. Blood 115:3835-3842.

Kumar A, Aakriti, Gupta V (2016) A review on animal models of stroke: an update. Brain Res Bull 122:35-44.

Liao Y, Smyth GK, Shi W (2014) featureCounts: an efficient general purpose program for assigning sequence reads to genomic features. Bioinformatics 30:923-930.

Li Q, Cheng Z, Zhou L, Darmanis S, Neff NF, Okamoto J, Gulati G, Bennett ML, Sun LO, Clarke LE, Marschallinger J, Yu G, Quake SR, Wyss-Coray T, Barres BA (2019) Developmental heterogeneity of microglia and brain myeloid cells revealed by deep single-cell RNA sequencing. Neuron 101: 207-223.e10.

Li X, Blizzard KK, Zeng Z, DeVries AC, Hurn PD, McCullough LD (2004)
Chronic behavioral testing after focal ischemia in the mouse: functional recovery and the effects of gender. Exp Neurol 187:94-104.

Liesz A, Suri-Payer E, Veltkamp C, Doerr H, Sommer C, Rivest S, Giese T, Veltkamp R (2009) Regulatory T cells are key cerebroprotective immunomodulators in acute experimental stroke. Nat Med 15:192-199.

Liesz A, Zhou W, Na SY, Hämmerling GJ, Garbi N, Karcher S, Mracsko E, Backs J, Rivest S, Veltkamp R (2013) Boosting regulatory T cells limits neuroinflammation in permanent cortical stroke. J Neurosci 33:1735017362.

Liesz A, Hu X, Kleinschnitz C, Offner H (2015) Functional role of regulatory lymphocytes in stroke facts and controversies. Stroke 46:1422-1430.

Llovera G, Hofmann K, Roth S, Salas-Pérdomo A, Ferrer-Ferrer M, Perego C, Zanier ER, Mamrak U, Rex A, Party H, Agin V, Fauchon C, Orset C, Haelewyn B, De Simoni MG, Dirnagl U, Grittner U, Planas AM, Plesnila $\mathrm{N}$, Vivien D, et al. (2015) Results of a preclinical randomized controlled multicenter trial (pRCT): anti-CD49d treatment for acute brain ischemia. Sci Transl Med 7:299ra121.

Llovera G, Liesz A (2016) The next step in translational research: lessons learned from the first preclinical randomized controlled trial. J Neurochem 139 [Suppl 2]:271-279.

Llovera G, Roth S, Plesnila N, Veltkamp R, Liesz A (2014) Modeling stroke in mice: permanent coagulation of the distal middle cerebral artery. J Vis Exp 89:e51729.

Love MI, Huber W, Anders S (2014) Moderated estimation of fold change and dispersion for RNA-seq data with DESeq2. Genome Biol 15:550.

Manwani B, Liu F, Xu Y, Persky R, Li J, McCullough LD (2011) Functional recovery in aging mice after experimental stroke. Brain Behav Immun 25:1689-1700.

Markram H, Toledo-Rodriguez M, Wang Y, Gupta A, Silberberg G, Wu C (2004) Interneurons of the neocortical inhibitory system. Nat Rev Neurosci 5:793-807.

Melbye P, Olsson A, Hansen TH, Søndergaard HB, Bang Oturai A (2019) Short-chain fatty acids and gut microbiota in multiple sclerosis. Acta Neurol Scand 139:208-219.

Meng H, Zhao H, Cao X, Hao J, Zhang H, Liu Y, Zhu MS, Fan L, Weng L, Qian L, Wang X, Xu Y (2019) Double-negative T cells remarkably promote neuroinflammation after ischemic stroke. Proc Natl Acad Sci U S A 116: $5558-5563$.

Morrison DJ, Preston T (2016) Formation of short chain fatty acids by the gut microbiota and their impact on human metabolism. Gut Microbes 7:189-200.

Murphy TH, Corbett D (2009) Plasticity during stroke recovery: from synapse to behaviour. Nat Rev Neurosci 10:861-872.

Nakamura YK, Janowitz C, Metea C, Asquith M, Karstens L, Rosenbaum JT, Lin P (2017) Short chain fatty acids ameliorate immune-mediated uveitis partially by altering migration of lymphocytes from the intestine. Sci Rep 7:11745.

Neumann J, Riek-Burchardt M, Herz J, Doeppner TR, König R, Hütten H, Etemire E, Männ L, Klingberg A, Fischer T, Görtler MW, Heinze HJ, Reichardt P, Schraven B, Hermann DM, Reymann KG, Gunzer M (2015) Very-late-antigen-4 (VLA-4)-mediated brain invasion by neutrophils leads to interactions with microglia, increased ischemic injury and impaired behavior in experimental stroke. Acta Neuropathol 129:259-277.

Offner H, Subramanian S, Parker SM, Afentoulis ME, Vandenbark AA, Hurn PD (2006) Experimental stroke induces massive, rapid activation of the peripheral immune system. J Cereb Blood Flow Metab 26:654-665.

Park J, Kim M, Kang SG, Jannasch AH, Cooper B, Patterson J, Kim CH (2015) Short-chain fatty acids induce both effector and regulatory T cells by suppression of histone deacetylases and regulation of the mTOR-S6K pathway. Mucosal Immunol 8:80-93.

Ransohoff RM, Engelhardt B (2012) The anatomical and cellular basis of immune surveillance in the central nervous system. Nat Rev Immunol 12:623-635.

Rehme AK, Grefkes C (2013) Cerebral network disorders after stroke: evidence from imaging-based connectivity analyses of active and resting brain states in humans. J Physiol 591:17-31.

Riccomagno MM, Kolodkin AL (2015) Sculpting neural circuits by axon and dendrite pruning. Annu Rev Cell Dev Biol 31:779-805.

Rosell A, Agin V, Rahman M, Morancho A, Ali C, Koistinaho J, Wang X, Vivien D, Schwaninger M, Montaner J (2013) Distal occlusion of the middle cerebral artery in mice: are we ready to assess long-term functional outcome? Transl Stroke Res 4:297-307. 
Roth S, Singh V, Tiedt S, Schindler L, Huber G, Geerlof A, Antoine DJ, Anfray A, Orset C, Gauberti M, Fournier A, Holdt LM, Harris HE, Engelhardt B, Bianchi ME, Vivien D, Haffner C, Bernhagen J, Dichgans M, Liesz A (2018) Brain-released alarmins and stress response synergize in accelerating atherosclerosis progression after stroke. Science Transl Med 10:11.

Salter MW, Stevens B (2017) Microglia emerge as central players in brain disease. Nat Med 3:1018-1027.

Schafer DP, Lehrman EK, Kautzman AG, Koyama R, Mardinly AR, Yamasaki R, Ransohoff RM, Greenberg ME, Barres BA, Stevens B (2012) Microglia sculpt postnatal neural circuits in an activity and complementdependent manner. Neuron 74:691-705.

Schindelin J, Arganda-Carreras I, Frise E, Kaynig V, Longair M, Pietzsch T, Preibisch S, Rueden C, Saalfeld S, Schmid B, Tinevez JY, White DJ, Hartenstein V, Eliceiri K, Tomancak P, Cardona A (2012) Fiji: an opensource platform for biological-image analysis. Nat Methods 9:676682.

Selvaraj UM, Stowe AM (2017) Long-term T cell responses in the brain after an ischemic stroke. Discov Med 24:323-333.

Shichita T, Sugiyama Y, Ooboshi H, Sugimori H, Nakagawa R, Takada I, Iwaki T, Okada Y, Iida M, Cua DJ, Iwakura Y, Yoshimura A (2009) Pivotal role of cerebral interleukin-17-producing gamma delta $\mathrm{T}$ cells in the delayed phase of ischemic brain injury. Nat Med 15:946-950.

Singh V, Roth S, Llovera G, Sadler R, Garzetti D, Stecher B, Dichgans M, Liesz A (2016) Microbiota dysbiosis controls the neuroinflammatory response after stroke. J Neurosci 36:7428-7440.

Singh V, Sadler R, Heindl S, Llovera G, Roth S, Benakis C, Liesz A (2018) The gut microbiome primes a cerebroprotective immune response after stroke. J Cereb Blood Flow Metab 38:1293-1298.

Smith PM, Howitt MR, Panikov N, Michaud M, Gallini CA, Bohlooly-Y M, Glickman JN, Garrett WS (2013) The microbial metabolites, short-chain fatty acids, regulate colonic T-reg cell homeostasis. Science 341:569-573.

Stephan AH, Barres BA, Stevens B (2012) The complement system: an unexpected role in synaptic pruning during development and disease. Annu Rev Neurosci 35:369-389.

Swidsinski A, Loening-Baucke V, Krüger M, Kirsch S (2012) Central nervous system and the colonic bioreactor: analysis of colonic microbiota in patients with stroke unravels unknown mechanisms of the host defense after brain injury. Intestinal Research 10:332.

Tan J, McKenzie C, Vuillermin PJ, Goverse G, Vinuesa CG, Mebius RE, Macia L, Mackay CR (2016) Dietary fiber and bacterial SCFA enhance oral tolerance and protect against food allergy through diverse cellular pathways. Cell Rep 15:2809-2824.

Thion MS, Low D, Silvin A, Chen J, Grisel P, Schulte-Schrepping J, Blecher R, Ulas T, Squarzoni P, Hoeffel G, Coulpier F, Siopi E, David FS, Scholz C, Shihui F, Lum J, Amoyo AA, Larbi A, Poidinger M, Buttgereit A, et al. (2018) Microbiome influences prenatal and adult microglia in a sexspecific manner. Cell 172:500-516.e516.

van de Wouw M, Boehme M, Lyte JM, Wiley N, Strain C, O'Sullivan O, Clarke G, Stanton C, Dinan TG, Cryan JF (2018) Short-chain fatty acids: microbial metabolites that alleviate stress-induced brain-gut axis alterations. J Physiol 596:4923-4944.

Villa A, Gelosa P, Castiglioni L, Cimino M, Rizzi N, Pepe G, Lolli F, Marcello E, Sironi L, Vegeto E, Maggi A (2018) Sex-specific features of microglia from adult mice. Cell Rep 23:3501-3511.

Walsh JT, Hendrix S, Boato F, Smirnov I, Zheng J, Lukens JR, Gadani S, Hechler D, Gölz G, Rosenberger K, Kammertöns T, Vogt J, Vogelaar C, Siffrin V, Radjavi A, Fernandez-Castaneda A, Gaultier A, Gold R, Kanneganti TD, Nitsch R, et al. (2015) MHCII-independent CD4(+) T cells protect injured CNS neurons via IL-4. J Clin Invest 125:699-714.

Wu Y, Dissing-Olesen L, MacVicar BA, Stevens B (2015) Microglia: dynamic mediators of synapse development and plasticity. Trends Immunol 36:605-613.

Xia GH, You C, Gao XX, Zeng XL, Zhu JJ, Xu KY, Tan CH, Xu RT, Wu QH, Zhou HW, He Y, Yin J (2019) Stroke Dysbiosis Index (SDI) in gut microbiome are associated with brain injury and prognosis of stroke. Front Neurol 10:397.

Zausinger S, Hungerhuber E, Baethmann A, Reulen H, Schmid-Elsaesser R (2000) Neurological impairment in rats after transient middle cerebral artery occlusion: a comparative study under various treatment paradigms. Brain Res 863:94-105.

Zhang Y, Chen K, Sloan SA, Bennett ML, Scholze AR, O'Keeffe S, Phatnani HP, Guarnieri P, Caneda C, Ruderisch N, Deng S, Liddelow SA, Zhang C, Daneman R, Maniatis T, Barres BA, Wu JQ (2014) An RNA sequencing transcriptome and splicing database of glia, neurons, and vascular cells of the cerebral cortex. J Neurosci 34:11929-11947.

Zhong S, Zhang S, Fan X, Wu Q, Yan L, Dong J, Zhang H, Li L, Sun L, Pan N, Xu X, Tang F, Zhang J, Qiao J, Wang X (2018) A single-cell RNA-seq survey of the developmental landscape of 720 the human prefrontal cortex. Nature 555:524-528. 